\title{
Intracellular Energy Variability Modulates Cellular Decision-Making Capacity
}

\author{
Ryan Kerr $\mathbb{1}^{1}$, Sara Jabbari $\mathbb{1}^{1}$ \& lain G. Johnston $\mathbb{D}^{2,3^{*}}$
}

Cells generate phenotypic diversity both during development and in response to stressful and changing environments, aiding survival. Functionally vital cell fate decisions from a range of phenotypic choices are made by regulatory networks, the dynamics of which rely on gene expression and hence depend on the cellular energy budget (and particularly ATP levels). However, despite pronounced cell-to-cell ATP differences observed across biological systems, the influence of energy availability on regulatory network dynamics is often overlooked as a cellular decision-making modulator, limiting our knowledge of how energy budgets affect cell behaviour. Here, we consider a mathematical model of a highly generalisable, ATP-dependent, decision-making regulatory network, and show that cell-to-cell ATP variability changes the sets of decisions a cell can make. Our model shows that increasing intracellular energy levels can increase the number of supported stable phenotypes, corresponding to increased decision-making capacity. Model cells with sub-threshold intracellular energy are limited to a singular phenotype, forcing the adoption of a specific cell fate. We suggest that energetic differences between cells may be an important consideration to help explain observed variability in cellular decision-making across biological systems.

Biological cells are faced with many decisions during their existence. Genetically identical single cells in a population choose different phenotypic strategies for survival; genetically identical cells in developing multicellular organisms make decisions to follow different developmental pathways, and hence towards one of a diverse range of possible phenotypes. Across organisms, gene expression variability leads to cell-to-cell variations in mRNA and protein levels in genetically identical cells, and can drive the generation of diverse phenotypes and strategies for survival ${ }^{1-5}$. The process of a cell assuming different functionally important fates from a range of phenotypic possibilities in response to or in anticipation of extracellular change, without genetic modifications, is known as a cellular decision ${ }^{6}$.

In multicellular organisms, phenotypic heterogeneity has been observed in a diverse range of cell types ${ }^{7}$ for a wide range of cellular decisions, from seed germination ${ }^{8-10}$ through the famous example of hematopoietic cell differentiation ${ }^{11,12}$, to mosaic development of retinal cells in the Drosophila eye ${ }^{13}$. Waddington's famous 'epigenetic landscape ${ }^{14}$ pictures these developmental decisions as bifurcating channels that a developmental 'ball' can roll down to select different possible cell fate decisions; bifurcations in the landscape correspond to multistability, where a cell can support distinct, differentiated cell fates. These repeated differentiation decisions allow, for example, human pluripotent stem cells to differentiate into all cell types in the human body ${ }^{15,16}$, while modern technology allows reprogrammed cells to move back 'up' the epigenetic landscape ${ }^{15,17}$. These cellular decisions are central to development and knowledge of their dynamics offers useful applications in medicine and fundamental biology ${ }^{18}$.

Single-celled organisms also embrace the advantages of diverse cell behaviours. Cells in their natural environment have to deal with the challenges presented by changes in extracellular conditions. These may include temperature changes, $\mathrm{pH}$ variability, nutrient limitation or, in some cases, the presence of antibiotics. To overcome such environmental fluctuations, genetically homogeneous cells can generate phenotypic diversity in order to increase the probability that some members of the population will survive ${ }^{19-21}$. This variability may enable a cell to support multiple, distinct phenotypes ${ }^{13,22,23}$ potentially helping a cell handle different environmental conditions; the resulting population heterogeneity may even increase the overall fitness of the species ${ }^{22,24}$.

${ }^{1}$ School of Mathematics \& Institute of Microbiology and Infection, University of Birmingham, Birmingham, United Kingdom. ${ }^{2}$ Department of Mathematics, Faculty of Mathematics and Natural Sciences, University of Bergen, Bergen, Norway. ${ }^{3}$ Alan Turing Institute, London, United Kingdom. *email: iain.johnston@uib.no 

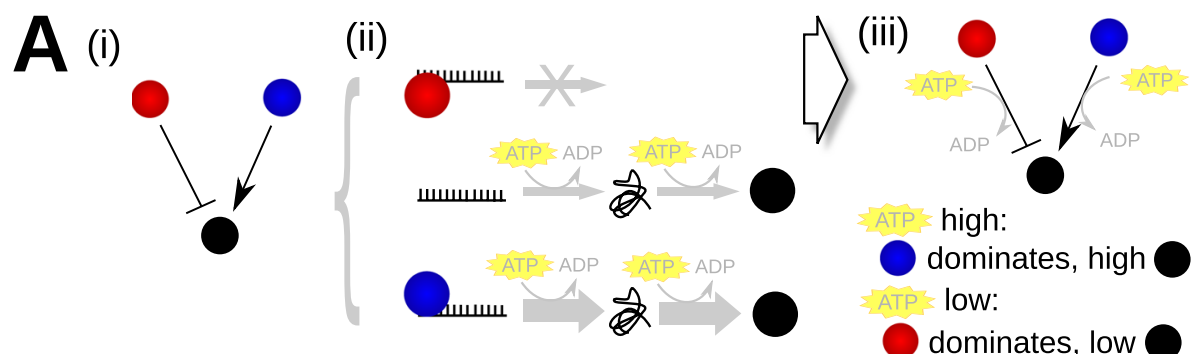

high:

dominates, high

low:
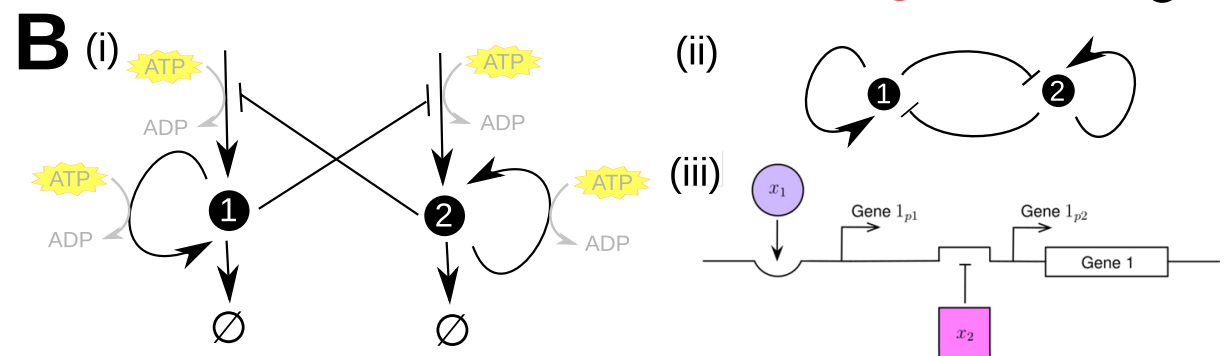

Figure 1. Energy dependence in model genetic architectures. (A) Energy dependence in gene regulation. In an example schematic regulatory network (i), genes have positive and negative regulatory interactions. The expression of these genes, and thus their regulatory interactions, rely on transcription and translation, processes with substantial ATP requirements (ii). The dynamics, behaviour, and states supported by a regulatory network are thus expected to be ATP-dependent (iii). In this example, when ATP levels are high, activation by a positive promoter (blue) may lead to high levels of expression of a downstream target (black). However, when ATP levels are low, there may be insufficient energy to support this increased expression level, and the system's behaviour is instead dominated by a repressive interaction (red). (B) The regulatory network for the general system considered in our model. The system involves two genes which self-activate and cross-repress, with both sets of processes being ATP-dependent (i). The energy dependence is often neglected in shorthand network representations of gene regulatory networks (ii). (iii) shows a possible arrangement of regulatory regions for one gene in the system that accomplishes this toggle-switch control.

In microbiology, diversity and cell decision-making underlie a range of biologically and medically important behaviours. Large-scale studies have revealed the generation of phenotypic diversity in yeast through noisy gene expression, potentially benefitting the population in varying and often stressful environments ${ }^{4,25}$. The decision for bacteria to sporulate into robust endospores is a survival mechanism used by diverse genera including Bacillus and Clostridium ${ }^{26}$, contributing to foodborne disease and food spoilage ${ }^{27,28}$. The decision to become a persister cell (a phenotype more tolerant to external stress, including antibiotics), is made in several bacterial species ${ }^{29-32}$ and can have a dramatic impact on the efficacy of medical treatments ${ }^{33-36}$.

Many of the mechanisms behind cellular decision making in eukaryotes and prokaryotes remain poorly understood, challenging our knowledge of fundamental biology and our ability to design cellular interventions to suit human purposes. Regulatory networks, representing the interactions between genes that govern these decisions, are often used to summarise our knowledge ${ }^{37}$. Typically, edges in a schematic network illustrate processes such as transcription and translation, and nodes represent genes. However, this coarse-graining can often omit a substantial amount of important detail. In particular, the fact that the processes represented by these edges are energy dependent (Fig. 1) is rarely considered. Transcription and translation require a substantial ATP budget ${ }^{38,39}$, so there exists a core energy dependence in the dynamics of gene regulatory networks, potentially affecting the decisions supported by a given cell.

This energy dependence is important because different cells, particularly in microbiology, can have substantially different levels of available energy. Energy variability has been observed within genetically-identical Escherichia coli cells in a population, where absolute concentrations of intracellular ATP were spread over at least half an order of magnitude, in a skewed distribution around $1.54 \pm 1.22 \mathrm{mM}^{40}$. Substantial intracellular ATP variability has also been observed in other branches of life, including yeast ${ }^{41}$, HeLa cells ${ }^{42}$ and plants ${ }^{43}$. Clearly, the dynamics, and thus potentially the outputs, of cellular decision-making networks may vary between cells due to these diverse energy levels.

Previous work has shown that energy availability can modulate the stability of decision-making circuits in eukaryotic cells ${ }^{44}$, supported by experimental observations linking, for example, mitochondrial content and quality (supporting high, stable ATP supply) to less differentiated cell outcomes ${ }^{16,45,46}$. However, the broader influence of energy variability on the behaviour of these circuits remains to be revealed, and a theoretical understanding of this potentially important modulator of cellular decision-making remains absent. Here, we seek to address this challenge using a theoretical approach that both provides highly generalisable understanding and circumvents the experimental difficulties in interrogating these complex systems. We use a mathematical representation of a regulatory network to model a genetic architecture observed as a decision-making motif across organisms. By including the effects of energy variability on physiological processes, we explore how the behaviour of the system 
will change when cell-to-cell variability exists in intracellular energy supply, and how this variability affects the ability of cells to make diverse decisions.

\section{Methods}

Regulatory architecture. We consider a well-known cellular decision-making architecture consisting of two genes, their respective protein products, and the regulation occurring through their interactions (Fig. $1 \mathrm{~B} ;{ }^{44,47}$ ). To study a general model of cellular decision-making we designate the genes 'Gene 1' and 'Gene 2', as displayed in Fig. 1B. Protein 1 and Protein 2 (expression levels $x_{1}$ and $x_{2}$ ) are expressed by Gene 1 and Gene 2 respectively, which then apply feedback control to the system through self-activation and cross-repression; both proteins also degrade. We define a cell fate as the level of these proteins at steady state.

We construct the governing ordinary differential equations (ODEs) with guidance from the literature ${ }^{47}$. We coarse-grain gene expression dynamics, avoiding an explicit representation of mRNA levels, and focussing on the levels of the protein products. The expression of each gene has contributions from self-activation (via the gene's product activating a conditional promoter) and from a constitutive promoter (which can be repressed by the other gene's product), Fig. 1B. For Gene $i$, the maximum expression level of the conditional promoter for Gene $i$ is $a_{i}$, and the basal expression level of the constitutive promoter is $b_{i}$. Each protein product is degraded with rate $k_{i}$. Overall we then obtain Eqs. (1) and (2) with initial conditions (3), previously studied by Huang et al. ${ }^{47}$, with $x_{1,0}, x_{2,0} \in \mathbb{R}^{+}$being some initial levels of proteins $x_{1}$ and $x_{2}$.

$$
\begin{gathered}
\frac{\mathrm{d} x_{1}}{\mathrm{~d} t}=a_{1} \frac{x_{1}^{n}}{\theta_{a_{1}}^{n}+x_{1}^{n}}+b_{1} \frac{\theta_{b_{1}}^{n}}{\theta_{b_{1}}^{n}+x_{2}^{n}}-k_{1} x_{1}, \\
\frac{\mathrm{d} x_{2}}{\mathrm{~d} t}=a_{2} \frac{x_{2}^{n}}{\theta_{a_{2}}^{n}+x_{2}^{n}}+b_{2} \frac{\theta_{b_{2}}^{n}}{\theta_{b_{2}}^{n}+x_{1}^{n}}-k_{2} x_{2}, \\
x_{1}(0)=x_{1,0}, \quad x_{2}(0)=x_{2,0} .
\end{gathered}
$$

The additive terms in Eqs. (1) and (2) reflect, from left to right, self-activation up to a maximum level of $a_{i}$, cross-repression down from a basal level of $b_{i}$, and degradation. The interaction processes are represented by Hill functions, with $n$ and $\theta$ parameters determining the steepness and inflection point of the sigmoidal curves, respectively; $\theta_{a_{i}}, \theta_{b_{i}}$ can be interpreted as the dissociation constants of the activator and inhibitor regulatory proteins to the promoter regions, respectively.

Huang et al. suggest a default example parameter set ${ }^{47}$, with $a_{i}=b_{i}=k_{i}=1, \theta_{a_{i}}=\theta_{b_{i}}=0.5, n=4(i=1,2)$. These parameters give rise to a tristable system, where, depending on initial conditions, the steady state can take one of three values. Interpreting these protein level values as cell fates, this tristability corresponds to three possible cell fates for an organism and therefore, for example, a wider decision-making landscape compared to a monostable system.

Energy dependence. Each step in transcription and translation requires energy in the form of ATP (Fig. 1), so we enforce that the rates of the corresponding gene expression processes in our model are dependent on an ATP concentration parameter. To this end, we first transform the parameters $a_{i}$ and $b_{i}$ to $a_{i} \rightarrow \lambda a_{i}$ and $b_{i} \rightarrow \lambda b_{i}$ $(i=1,2)$ with $\lambda$ being a function of intracellular ATP level. For simplicity and generality, we ignore the possible energy dependence of degradation as being of lower magnitude than these constructive processes. We will usually consider $\lambda$ values between 0 and 1, reflecting the modulation of transcription rates from a theoretical maximum value (when ATP supply is not rate-limiting) to zero (in the absence of any available ATP supply), but consider relaxation of this scale later.

The rates of biophysical processes depend on corresponding free energy availability, a complicated quantity that for gene expression strongly depends on cellular ATP:ADP ratio, as well as other metabolic factors. Typical ATP:ADP ratio values have been shown to vary between $2-3$ in mouse pancreatic $\beta$-cells ${ }^{48}$, and $19 \pm 4$ in wild-type E. coli cells ${ }^{49}$; variability has also been observed within and between different plant cells and cellular components ${ }^{50}$. To model this complex quantity and make it transferable across different biological species, we use a parameter $A^{*}$ to reflect a ratio of a cell's free energy availability to a maximum possible value. This sets the upper and lower limits of $A^{*}$ to be 0 and 1 for each cell, and values of $A^{*}$ can be mapped to, for example, specific ATP concentrations for a given biological system of interest (Supplementary Fig. S1-S2).

To model the dependence of transcription rate $\lambda$ on energy availability $A^{*}$, we follow das Neves et al..$^{38}$, who found a sigmoidal relationship between the total transcription rate in a cell and ATP concentration. As we are primarily concerned with the ATP dependence of gene expression, we modelled $\lambda$ with a sigmoidal curve, yielding monotonically increasing rates as energy increases (adoption of a linear rather than a sigmoidal relationship between scaled energy and dynamic rates did not change our qualitative observations, Supplementary Fig. S1). Specifically, we use Eq. (4), where $s_{1}=16, s_{2}=-8$ are chosen to produce the monotonically increasing curve for $\lambda\left(A^{*}\right)$ in Supplementary Fig. S2.

$$
\lambda\left(A^{*}\right)=\frac{1}{1+e^{-\left(s_{1} A^{*}+s_{2}\right)}} .
$$


For biological reference, Supplementary Fig. S2 displays the known range of intracellular ATP concentration of E. coli $\left(1.54 \pm 1.22 \mathrm{mM}^{40}\right)$. By assuming a constant sum of ATP and ADP concentrations within an E. coli cell, as observed by Jensen and Michelson ${ }^{49}$ for wild-type and atp mutant cells, an increase in ATP concentration corresponds to an increase in free energy availability by changing the ATP:ADP ratio. At the lower bound of ATP concentration, $320 \mu \mathrm{M}, \lambda$ is small, equating to a low maximum expression level. For the upper bound, $2760 \mu \mathrm{M}$, $\lambda$ is at its maximum value and represents maximal expression level. Concentrations below $320 \mu \mathrm{M}$ are outside the detected E. coli ATP range ${ }^{40}$, so we take this to be the critical intracellular energy to continue as a living cell and therefore may represent non-living or dying cells. Overall we thus obtain:

$$
\begin{aligned}
& \frac{\mathrm{d} x_{1}}{\mathrm{~d} t}=\lambda\left(A^{*}\right) a_{1} \frac{x_{1}^{n}}{\theta_{a_{1}}^{n}+x_{1}^{n}}+\lambda\left(A^{*}\right) b_{1} \frac{\theta_{b_{1}}^{n}}{\theta_{b_{1}}^{n}+x_{2}^{n}}-k_{1} x_{1}, \\
& \frac{\mathrm{d} x_{2}}{\mathrm{~d} t}=\lambda\left(A^{*}\right) a_{2} \frac{x_{2}^{n}}{\theta_{a_{2}}^{n}+x_{2}^{n}}+\lambda\left(A^{*}\right) b_{2} \frac{\theta_{b_{2}}^{n}}{\theta_{b_{2}}^{n}+x_{1}^{n}}-k_{2} x_{2} .
\end{aligned}
$$

Time-dependent and steady state numerical solutions of Eqs. (5) and (6), with Eq. (4), were generated in Python 3.6.5 using the integrate.odeint and optimize.root functions within the scipy module ${ }^{51}$; integrate.odeint is a stiff or non-stiff solver for a system of first-order ordinary differential equations which implements a Backward Differentiation Formula (stiff) or predictor-corrector (non-stiff) method, and optimize.root solves the system of nonlinear equations using a modification of the Levenberg-Marquardt algorithm. Prescribed initial conditions covered a grid of $x_{1}$ and $x_{2}$ at equally spaced points when calculating numerical solutions; bifurcation diagrams and heatmaps were constructed using the numerical solutions. In the case where multiple degenerate solutions are found, attractor quantity was confirmed using both Maple (2019) and Matlab (R2018a; Mathworks). All scripts used for this study are openly accessible through https://github.com/StochasticBiology/ energy-variability-decision-making.

For each parameter set, the stable steady states of the system correspond to cell fates. We do not consider unstable or metastable steady states to represent cell fates as small perturbations in expression due to noise will move the system towards a stable point; we consider both unstable and metastable steady states as 'functionally unstable' in our study. If multiple stable states exist, the level of proteins $x_{1}$ and $x_{2}$ at each steady state determine the different phenotypes a cell can generate, as shown by Huang et al. ${ }^{47}$; in their study, high $x_{1}$ and low $x_{2}$ corresponded to a progenitor cell differentiating into an erythroid cell, rather than a myeloid cell.

As we are modelling the observed interactions in Fig. $1 \mathrm{~B}$ rather than biochemical interactions, the analysis of our results considers the qualitative behaviour rather than observing quantitative results. From this point, we set $a=a_{1}=a_{2}, b=b_{1}=b_{2}, k=k_{1}=k_{2}, \theta_{a}=\theta_{a_{1}}=\theta_{a_{2}}, \theta_{b}=\theta_{b_{1}}=\theta_{b_{2}}$ and each section will explicitly state the fixed and varied parameters for the work that follows.

Accession codes. No data were generated during this project.

\section{Results}

Our model system generally exhibits dynamic behaviour that, starting from some initial condition $\left(x_{1,0}, x_{2,0}\right)$, converges to a particular steady state characterised by values $\left(x_{1}, x_{2}\right)$. A limited number of these steady states exist for a given parameterisation of the system; these are attractors, so called because initial conditions are 'attracted' towards these stable states. A range of initial conditions will converge to the same attractor; this range of $\left(x_{1,0}, x_{2,0}\right)$ values is the attractor basin of that attractor.

We view distinct attractors, with distinct patterns of protein levels, as distinct cell fates. The attractor basin of each corresponds to the range of cell states that will return to that cell fate. This range makes each fate robust to fluctuations: for example, a small change in the level of one protein in one attractor state will likely still fall within that attractor's basin, so the system will return there.

In the following sections, we consider the number and properties of distinct cell fates (attractors) supported by the system at different energy levels. As we increase energy levels we often observe bifurcation, where one stable state transitions to two new states (increasing the number of options for cellular decision making). We also consider the "difficulty" of switching between attractors (moving from one cell fate to another); in biology this can be accomplished through noisy or controlled external influences ${ }^{52-54}$.

Intracellular energy budget modulates cellular decision-making landscapes. We first sought to understand how increasing energy availability changes the number of decision-making options available to the cell. To this end, we explored the bifurcation behaviour of the steady-state protein levels in our model as we changed energy availability. As our parameterisation for now imposes a symmetric structure on the phase space of steady-state protein levels, we begin by simply using the diversity of $x_{2}$ levels to illustrate the range of attractors present in the system for given energy levels (example in Supplementary Fig. S3). Throughout this section, $\theta_{a}, \theta_{b}$ and $n$ are fixed at their default values, and parameters $a, b$ and $k$ are varied.

We explored the emergence of different attractors (hence, the emergence of different cell fates that can be decided between) with energy level $A^{*}$ under a variety of different parameterisations. A clear general trend emerged, whereby the number of distinct attractors supported by the system increases with increasing energy availability (Fig. 2). There is thus an increased diversity of stable protein states, and hence an increased number of options for cellular decisions, if energy levels are higher. The separation of these attractors also increased with increasing energy, with the attractor basin associated with the intermediate attractor becoming wider, in 
(a) $a=0.00$

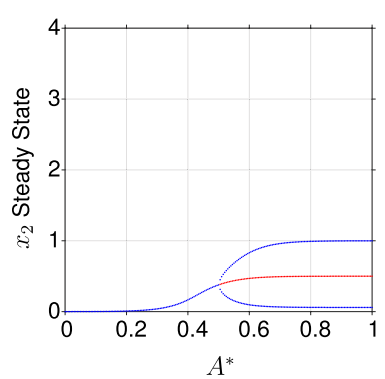

(e) $b=0.25$

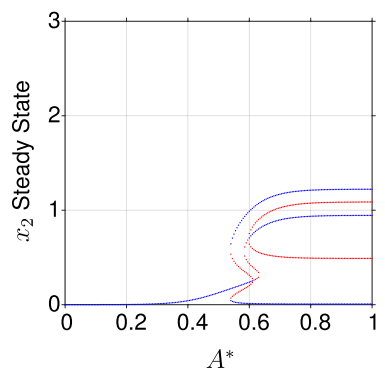

(i) $k=0.50$

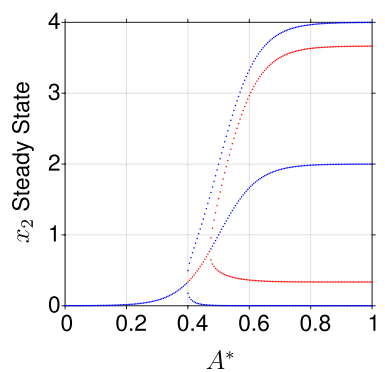

(b) $a=1.25$

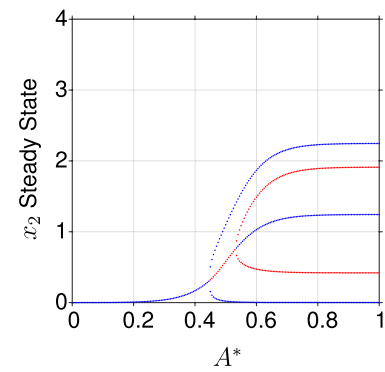

(f) $b=0.50$

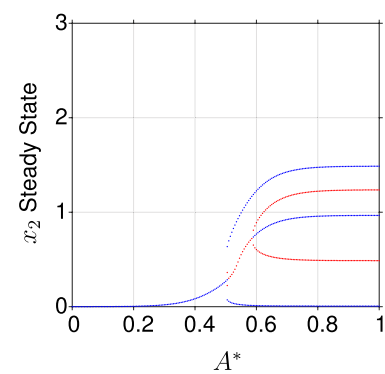

(j) $k=1.25$

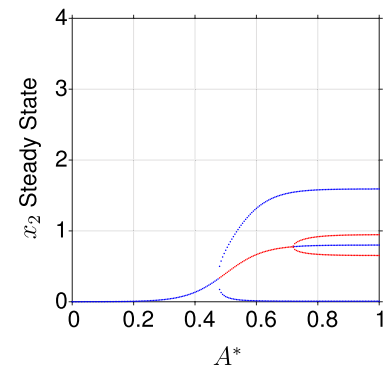

(c) $a=1.75$

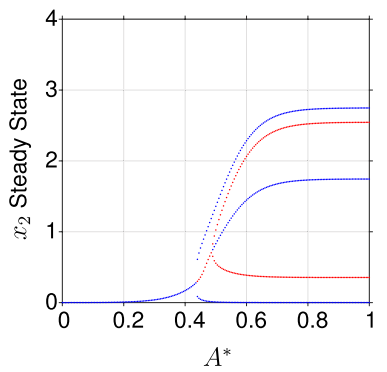

(g) $b=0.75$

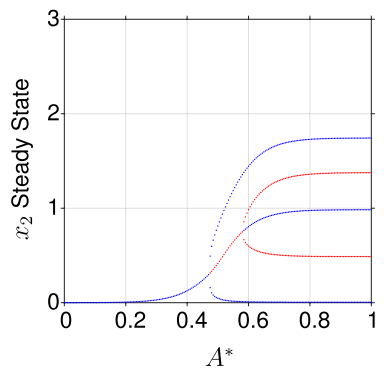

(k) $k=1.50$

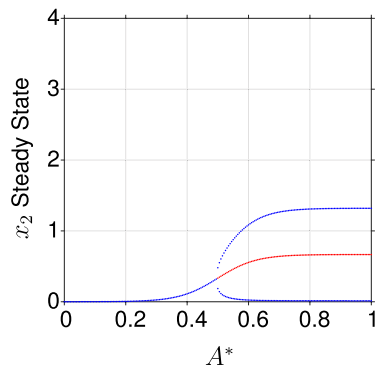

(d) $a=3.00$

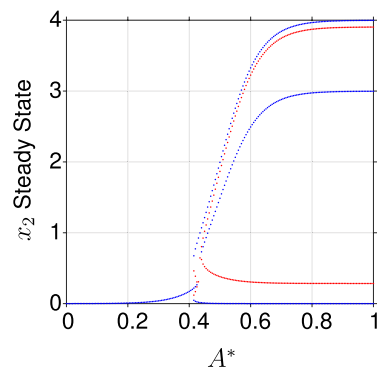

(h) $b=1.50$

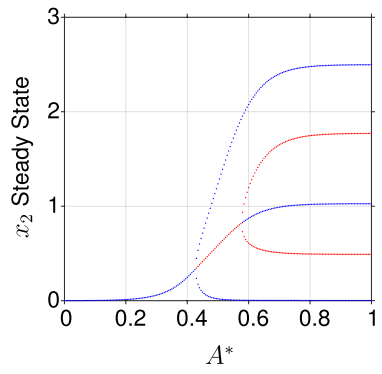

(I) $k=3.00$

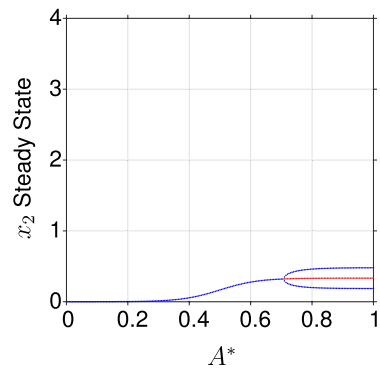

Figure 2. Attractor basins with increasing energy availability $A^{*}$. Bifurcation diagrams show increasing values for: (a-d) maximum expression level from conditional promoter; (e-h) constitutive promoter level; (i-1) degradation. Plots display $x_{2}$ values for stable steady states (blue circles) and unstable steady states (red circles) over $A^{*} \in[0,1]$ in steps of size $5 \times 10^{-3}$.

agreement with Johnston et al. and Huang et al. ${ }^{44,47}$. This suggests a biological stabilisation of states characterised by intermediate protein levels (see Fig. 2).

The quantitative properties of these attractor states vary with model parameters. Increased promoter activity $a$ and $b$ has the effect of increasing the separation of protein levels in distinct states, likely due to a simple elevation of maximum level. Correspondingly, increased degradation activity $k$ has the opposite effect, diminishing the differences between distinct states. In particular, high values of $k$ prevent the emergence of tristability, limiting the system to bistability even at high energy levels (Fig. 2(k,l)).

The bifurcation dynamics display some subtle variation in different cases. For example, at high levels of $a$ (the maximum expression level of the conditional promoter), increasing energy availability drives the system through re-entrant behaviour, where the number of attractors (decision options) runs from 1 to 3 then down to 2 before returning to 3 (see Supplementary Figs. S4 and S5 for detailed views of this behaviour). We shall see below (for example in Figs. 3 and 4) that similar behaviour is also observed in other regions of parameter space.

Generally, a single stable branch exists below an $A^{*}$ threshold. Biologically this implies that a single cell fate exists below an intracellular energy level, restricting the decision-making ability of a cell under this architecture. Once intracellular energy budget exceeds a threshold, multiple steady states are supported, enabling cellular decision-making. All our results demonstrate that, for our decision-making architecture, an increase in $A^{*}$ generally has the effect of accumulating stable branches in the bifurcation diagrams. This means a broader decision-making landscape for a cell when it has high intracellular energy budget. If degradation, $k$, is too high the central stable attractor does not exist, and the system is limited to bistability. Therefore, the decision-making capabilities of a cell are reduced if the degradation level is sufficiently high. Re-entrant stable steady state behaviour exists for certain parameter sets, suggesting a potential optimum intracellular energy range for phenotypic diversity. This may give a cell an optimal range of intracellular energy levels to support an increased variety in cell phenotypes, enabling, for example, superior adaption to extracellular environmental changes. This behaviour could also have negative consequences for a cell due to phenotypes becoming unsupported with slight variances in energy, forcing a cell to establish a different phenotype which, due to being a time dependent process, may not be performed quick enough to tolerate an environmental change. 


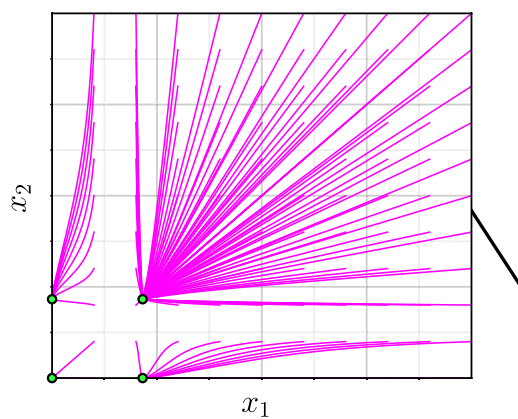

$x_{1}$

\begin{tabular}{ll|l|l|l|l|l|l|l|l|l|l|l|l|}
\hline 4 & 3 & 3 & 3 & 3 & 3 & 3 & 3 & 3 & 3 & 3 & 3 & 3 \\
\hline & 3 & 3 & 3 & 3 & 3 & 3 & 3 & 3 & 3 & 3 & 3 & 3
\end{tabular}

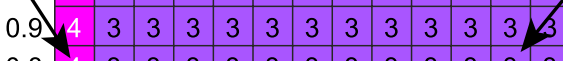
\begin{tabular}{llll|l|l|l|l|l|l|l|l|l|l|l|}
0.8 & 4 & 3 & 3 & 3 & 3 & 3 & 3 & 3 & 3 & 3 & 3 & 3 & 3 \\
\hline
\end{tabular}

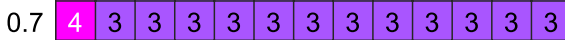

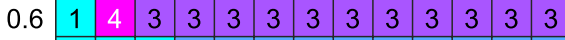

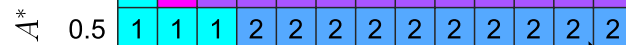
$0.4 \begin{array}{lllllllllllllll}0.4 & 1 & 1 & 1 & 1 & 1 & 1 & 1 & 1 & 1 & 2 & 2 & 2\end{array}$

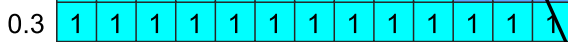
\begin{tabular}{llllllllllllllll|}
0.2 & 1 & 1 & 1 & 1 & 1 & 1 & 1 & 1 & 1 & 1 & 1 & 1 & 1 \\
\hline
\end{tabular} \begin{tabular}{l|llllllllllllll}
0.1 & 1 & 1 & 1 & 1 & 1 & 1 & 1 & 1 & 1 & 1 & 1 & 1 & 1 \\
\hline
\end{tabular}

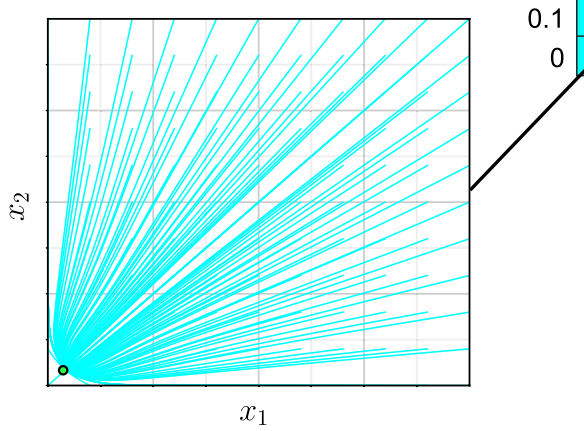

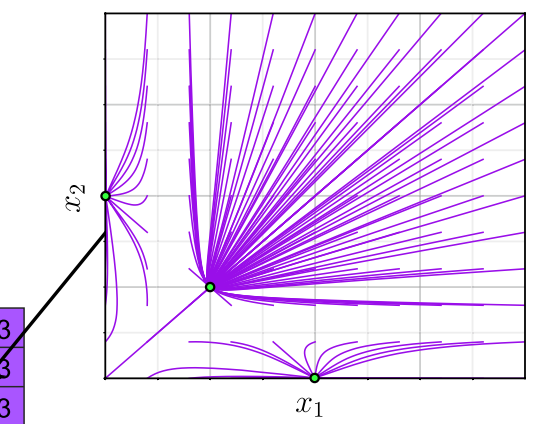

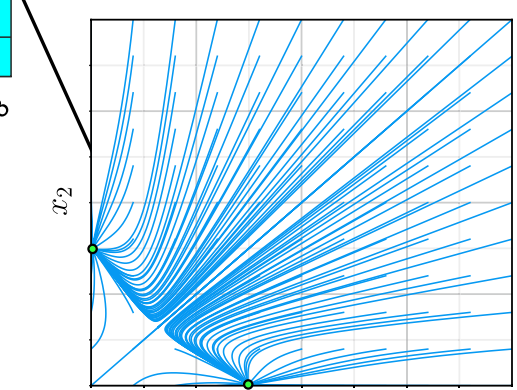

$x_{1}$

Figure 3. Decision-making capacity depends on cellular energy and dynamic parameters. Heatmap shows number of distinct attractors for $a=1, b \in[0,3]$ and $A^{*} \in[0,1]$, with all remaining parameters fixed at their default values. Inset figures present examples of the attractor landscape with solution trajectories (coloured) and stable steady states (green circles with black circumference). The 4 categories are, when viewed in portrait orientation, 1 stable steady state (bottom left, turquoise), 2 stable steady states (bottom right, blue), 3 stable steady states (top right, purple) and 4 stable steady states (top left, pink).

In Fig. 3 we show this 'phase portrait' of attractor landscapes for varying energy $A^{*}$ and constitutive expression $b$, along with example structures of the corresponding attractor basins. The general observation of increasing number of fate options with energy holds throughout. There is a separatrix line in this phase portrait between regions supporting a single attractor basin and those supporting more than one. For a given dynamic parameter, this separatrix is crossed as energy availability increases, then further increase has the effect of expanding the attractor basin associated with the state where both genes are expressed symmetrically (as in Johnston et al. ${ }^{44}$ ).

What is the physical intuition behind these structural changes with energy and dynamic parameters? Start with the low activity, low energy case. Here there is simply not enough cellular 'production capacity' - the ability to express genes - to allow dynamic signalling interactions between gene products. The system is limited to low levels of expression for both products, favouring a single, low-expression state.

As we now increase $b$, the basal expression rate increases, and the high coefficient associated with the cross-repression term induces very high antagonism - any balance between the gene products is highly unstable. The system invests all available energy getting to either one state or another. The low energy levels and correspondingly low expression levels mean that 'small number' effects are important: any small perturbations from symmetric expression will be amplified to favour one gene over the other.

In the alternative case, where we have low basal expression but high energy, we have substantial 'production capacity' that can now support an energetically more demanding intermediate state. Higher expression levels mean that fluctuations can be buffered and intermediate states are more stable. Here, there is constant tension between genes, requiring high energy levels; the fixed states are also still supported. In order to favour either gene product we need enough initial protein to jump-start the system (otherwise we reach the trivial zero or near-zero attractor).

This latter case remains true in the final case of high energy and high basal expression, except here the zero or near-zero attractor is unstable because from here, the substantial 'production capacity' means that some protein can always be produced, which is likely to drive the system towards one of the non-trivial attractors.

We next explored this behaviour varying both promoter levels $a$ and $b$ together (Fig. 4). At low values of the conditional promoter activity $(a \leq 0.5)$ the system is restricted to mono- or bistability for all values of $b$ and a single stable attractor exists below an $A^{*}$ threshold. High values of $a$ support the greater diversity of stable states previously seen in Fig. 3, however the attractor landscape changes minimally for higher $a$ (Fig. 4(e,f)). For high $a$ 
(a) $a=0.00$

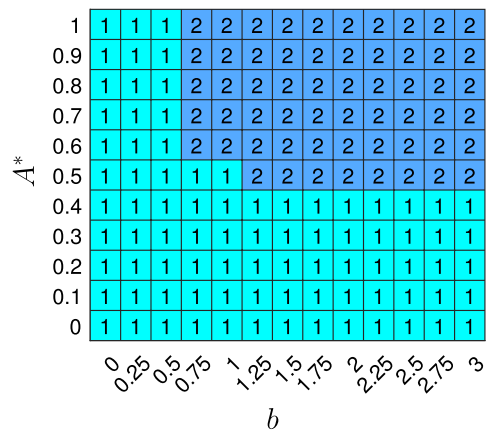

(d) $a=1.50$

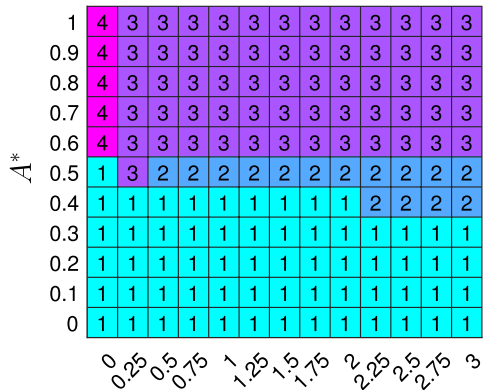

$b$ (b) $a=0.50$

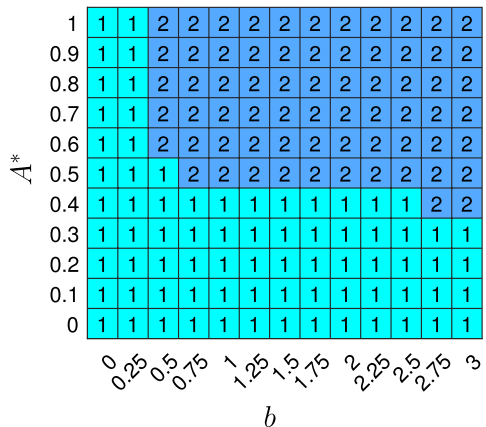

(e) $a=2.00$

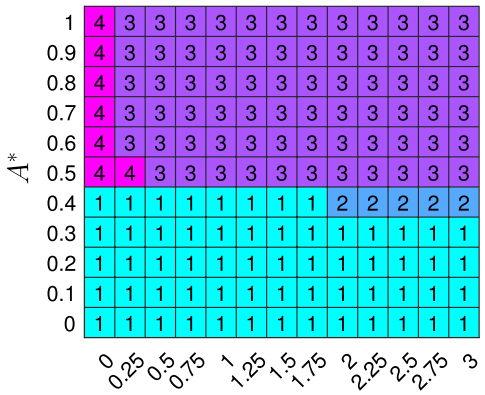

$b$ (c) $a=1.00$

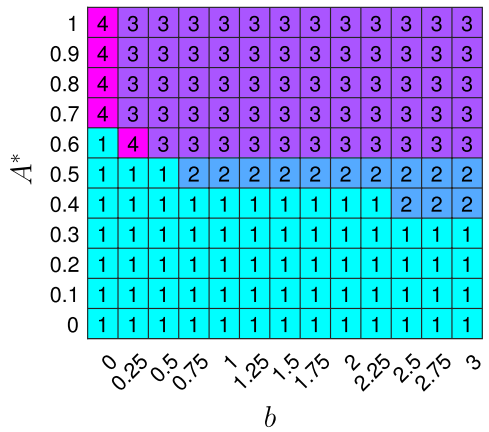

(f) $a=3.00$

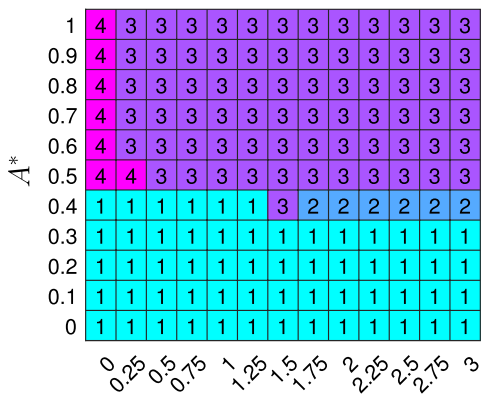

b

Figure 4. Decision-making landscapes depend on energy availability and dynamic rate parameters. Panels (a-f) display heatmaps of number of distinct attractors for 6 increasing values of $a$, when $n=4$. Each panel exhibits the number of stable steady states for combinations of $b \in[0,3]$ and $A^{*} \in[0,1]$. Throughout $(\mathbf{a}-\mathbf{f})$, all parameters, except $a, b$ and $A^{*}$, are fixed at their default values.

and some values of $b$, we again see the re-entrant behaviour from the bifurcation diagrams (Supplementary Figs. S4 and S5), with the 4-attractor state supported only at some intermediate values of energy availability. Illustrations of the nature of these re-entrant steady states are given in Supplementary Figs. S6 and S7, for increasing values of $A^{*}$ and $b$ respectively.

This re-entrant behaviour for increasing values of $A^{*}$ or $b$ (Supplementary Figs. S4-S7) merits further analysis. The reduction from 4 to 3 stable states with increasing energy is likely due to the destabilisation of the near-zero attractor (Supplementary Fig. S6), where expression levels of both genes is low. Above an energetic threshold, a baseline of production will be constantly occurring, and if the activity $a$ of the conditional promoter is high enough, any small increases in protein level can rapidly self-amplify and drive the system to an attractor with higher expression of protein products. The 4-attractor system will then only be supported at low $b$ and sufficiently low energies, as we observe. When the number of attractors (decision options) runs from 1 to 3 then down to 2 before returning to 3, the central stable attractor is destabilised as energy increases (Supplementary Fig. S7). This is likely due to a higher contribution from the constitutively expressed promoter as a proportion of the overall expression when energy increases, enhancing the antagonism, increasing the overall expression of both proteins and therefore driving the system to one state or the other as the central state cannot be sustained.

In summary, when $a$ is small (Fig. 4(a,b)), the system is limited to mono- or bistability, which may equate to a limited diversity in phenotypic possibilities for a cell. Increasing $a$ lowers the separatrix and decreases the $A^{*}$ threshold for tristability. Biologically, this suggests that for our decision-making architecture, as the maximum expression level of the promoter under activator control increases, less energy is required for an expansion in a cell's decision-making landscape. Independent of the value of $a$ and $b$, a singular stable steady state always exists at low energy, in theory limiting a cell to generating a singular phenotype.

Cooperativity effects on system behaviour. We next asked how these relationships between attractor basin structure (number of decision options) and energy depends on the cooperative nature of our model interactions (represented by the Hill coefficient $n$ in our governing equations). We found that decreasing the Hill coefficient to $n=3$ (Supplementary Fig. S8) led to some minor rearrangements of the phase portrait, decreasing to $n=2$ (Supplementary Fig. S9) does not display any re-entrant behaviour, while the quasi-linear case of $n=1$ (Supplementary Fig. S10) removed any diversity in attractor basin structure for the parameters we consider.

Therefore, if there is noncooperative binding $(n=1)$ of the proteins, the number of stable states is limited to one (Supplementary Fig. S10). This would relate to no decision-making capability for any cell, independent of intracellular energy level or gene expression levels, in the regimes we are considering. In contrast, if cooperativity is reduced from $n=4$ (default value, Fig. 4 ) to $n=3$ or $n=2$ (Supplementary Fig. S8 or S9 respectively), the 
observed behaviour is qualitatively similar, suggesting that a limited degree of nonlinearity in cellular interactions is sufficient to support decision-making and that energy dependence remains important even in these limited cases.

Regulatory protein binding strength can limit system behaviour. To understand how the binding strength of regulatory proteins affects the decision-making abilities of a cell, the influence of Hill parameters $\theta_{a}$ and $\theta_{b}$ on attractor basin structure was also analysed (Supplementary Fig. S11). $\theta_{a}$ and $\theta_{b}$ can be interpreted as the dissociation constants of the activator and inhibitor regulatory proteins to the promoter regions, respectively. Increasing $\theta_{a}\left(\theta_{b}\right)$ corresponds to decreasing the binding affinity of the corresponding protein to the relevant promoter site, thus requiring more protein to achieve the equivalent levels of activation (inhibition).

Changing $\theta_{a}$ changes the $A^{*}$ threshold required for tristability; there is a 'sweet spot' in $\theta_{a}$ that supports tristability at higher $A^{*}$ values. This behaviour is not observed for $\theta_{b}$; for low $\theta_{b}$, single, double, and triple stable states are observed at different energy levels, while for high $\theta_{b}$ the system is limited to a single attractor.

Hence, the relationship between energy availability and decision-making capacity of the system depends differently on the interactions encoded by $\theta_{a}$ and $\theta_{b}$ (Supplementary Fig. S11). There is an optimal range of $\theta_{a}$ for tristability, and outside of this range it is more probable for the system to be bistable, but it can also be limited to monostability. This suggests a cell requires a stronger activator binding strength to be less sensitive to changes in the number of activator proteins modulating expression levels. The optimal range for tristability is increased when parameter $\theta_{b}$ is varied. A cell would be less sensitive to changes in the number of inhibitor proteins modulating expression for both weak and strong binding strengths of the inhibitor protein. When the inhibitor binding strength becomes too weak the system is limited to a single stable state, removing the decision-making ability for a cell.

External ATP effects on decision-making landscapes. We next asked how the presence of external ATP sources (for example, from the environment, other cells, or a host cell in the case of a parasite) affected decision-making behaviour in our model. The effect of uptake of external ATP is to raise the baseline level of energy available for transcription ${ }^{38}$. We capture this by enforcing the sigmoid function $(\lambda)$ to be nonzero at $A^{*}=0$, and allowing its maximum to surpass 1 (Supplementary Fig. S12). This shift models an externally available ATP source for a cell. We found that external ATP supply shifted the profile of supported phenotypic states towards those with more stable phenotypes (Supplementary Fig. S13), suggesting that external ATP enables the cell to enter a higher-decision state-space more easily (for example, compare Fig. 4(c) and Supplementary Fig. S13(c)).

Stable steady-state transitions. The transition between phenotypes can be analysed by observing the 'difficulty' of moving between attractors at each energy level. Here, we consider the minimum biochemical perturbation required to transition from one extreme attractor state to another, by calculating the distance required to transition directly or indirectly between extreme attractor basins. This distance qualitatively corresponds to the magnitude of an intrinsic fluctuation, or an externally induced change, in gene expression levels required to induce a permanent shift in phenotype. Specifically, we compute the minimum of the following quantities: (i) the shortest distance in $\left(x_{1}, x_{2}\right)$ space between the high- $x_{1}$ attractor and the high- $x_{2}$ basin; (ii) the sum of shortest distances between the high- $x_{1}$ attractor and an intermediate basin and the shortest distance from that intermediate attractor to the high $-x_{2}$ basin. In (ii), an indirect transition within the 3 or 4 attractor landscape can occur through a move into an intermediate attractor basin, relaxing towards the intermediate attractor point, and then a movement into the second extreme attractor basin. This distance is only calculated for landscapes with 2 or more attractors, as a monostable system cannot transition.

The easiest transitions (shortest distances) occur as soon as the attractor landscape transitions from monostable to multi-stable (Fig. 4 and Supplementary Fig. S14), where extreme attractors are closest with little intermediate volume of phase space. The most difficult transitions (hence, most stable phenotypic states) appear for high values of the conditional promoter activity $(a=3)$ where attractors in the landscape are spread apart (see Supplementary Fig. S14(f)). We consistently observe the most stable phenotypic states for large $b$, however, for intermediate $a$ values (Supplementary Fig. S14(c,d)) the transition difficulty at large $b$ values is reduced compared to low and high $a$ (Supplementary Fig. S14(b,f) respectively).

Therefore, increasing energy availability both increases the number of phenotypic choices supported by our model architecture and stabilises the corresponding states (Fig. 4 and Supplementary Fig. S14). For a bistable system, moderate $A^{*}$ enables easier transitions, and an increase in energy availability shifts the transitions to be considerably more difficult, stabilising phenotypes. In a tristable landscape, the transition 'difficulty' is initially similar to the bistable case, but an increase in $A^{*}$ does not alter the transition distance to match that of the bistable landscape, unless $a$ is very high, where transitions become considerably more difficult.

\section{Discussion}

We have used a simple and general regulatory model, representative of the core of several known decision-making motifs ${ }^{47,55-58}$, to explore how the decision-making capacity of a cell depends on the energy available to fuel the processes involved in this decision making. Our theory predicts that, across a broad range of biological contexts, differences in cellular energy levels will cause differences in the dynamics and stable outcomes of cellular decision-making, via modulation of the expression rates of interacting regulatory genes. More precisely, increased ATP levels both support the ability to transition into multiple stable states, and increase the separation between the attractors as energy increases, stabilising the decisions that can be made (Fig. 2). Concurrently, without a sufficient energy supply, decision-making circuits lose the capacity to select different phenotypes (Figs. 2-4). Higher energy levels allow, and stabilise, separate and intermediate states. 
Our findings - that the fate choices supported by decision-making architectures depend on available energy - exist independently of the other metabolic requirements of given precursor or descendant cell types. It is fundamentally the influence of ATP variability on the dynamics of regulatory motifs that can cause this general dependence, which exists in addition to specific metabolic budgets and responses of the cell types in question.

The architecture we consider (Fig. 1B) can describe the behaviour of many naturally-occurring decision-making circuits, and our findings that energy availability influence this behaviour thus provide testable predictions for this class of systems. Accordingly, our prediction that phenotypic decisions depend on ATP is already supported by several experimental observations, discussed below. Our hypothesis can further be readily tested by, for example, tracking prokaryotic phenotype or eukaryotic fate decisions in a culture exposed to the same stimulus but titrated with different external concentrations of ATP.

This observation that different levels of ATP may induce different cellular behaviours comes as increasing cell heterogeneity in ATP levels is being experimentally characterised. Intracellular ATP has been quantified to vary between $0.32-2.76 \mathrm{mM}$ in a bacterial population ${ }^{40}, 3.7-3.9 \mathrm{mM}$ in HeLa cells ${ }^{42}, 3-4 \mathrm{mM}$ in living yeast cells ${ }^{41}$ and over a several-fold change occurs in different tissues of a plant ${ }^{43}$. The connection between energy variability and cellular decision-making has also been discussed previously ${ }^{31,59}$, further supporting the hypothesis that energy diversity may modulate the number of regulatory states supported by a cell, playing an important causal role in inducing variability in cellular decision-making.

Setting $a_{1}=a_{2}=0$ reduces the system to consist of a bistable switch with cross repression, known as a toggle-switch. This motif is found at the core of several known decision-making architectures throughout biology, with an extensive body of associated literature ${ }^{60-67}$. This motif famously occurs in the $\lambda$-bacteriophage switch from lysogenic to lytic state in Escherichia coli. The lysis-lysogen decision can generally be described as being determined by two genes, $c I$ and $c r o$, which mutually cross-repress through their products; the outcome of high $c I$ (cro) expression is cro $(c I)$ repression and $c I$ (cro) transcription, resulting in the lysogenic (lysis) state ${ }^{68-71}$. The switch from lysogenic to lytic state occurs when the $c I$ product is cleaved by RecA, a result of the response to DNA damage, derepressing cro, increasing Cro production and turning off $c I^{68}$.

The cross-repressing motif is observed in eukaryotic development ${ }^{56,58}$, where, for example, cross-repressing mammalian transcription factors $\mathrm{Cdx} 2$ and Oct4 govern the branch point between the trophectoderm and the inner cell mass in pluripotent embryonic cells ${ }^{72}$, and the bistable 442 MAPK/Cdc2 system controls maturation of the Xenopus oocyte ${ }^{73}$. The situation $a \neq 0$, including self-promotion in the regulatory motif, describes more architectures such as B cells promoting an antibody class switch ${ }^{74}$, gene regulatory networks with slow promoter kinet$\mathrm{ics}^{75}$ and cell-fate development and differentiation in eukaryotic cells ${ }^{55,57,76-79}$. A famous example is the GATA-1-PU.1 system controlling hematopoietic stem cell differentiation, studied theoretically ${ }^{47,75,80-83}$ (with some consideration of the influence of energy variability ${ }^{44}$ ). The relationship between pluripotent stem cell behaviour and energy metabolism has also been extensively studied ${ }^{16}$. Culturing human embryonic stem cells (ESCs) in low oxygen (suppressing oxidative phosphorylation and thus reducing energy production) prevents differentiation $^{84}$. Differentiation of pluripotent stem cells (PSCs) to cardiomyocytes requires a high oxidative phosphorylation capacity ${ }^{85}$, and human PSCs maintain higher mitochondrial membrane potential $\left(\Delta \Psi_{m}\right)$ than their differentiated descendants ${ }^{86}$. In mouse ESCs, cells with high $\Delta \Psi_{m}$ remained in a pluripotent state while cells with low $\Delta \Psi_{m}$ largely adopted a single differentiated state ${ }^{46}$. These observations are all compatible with our prediction of reduced decision-making capacity at low energy levels due to the energy dependence of regulatory networks (although the different metabolic requirements of different cell types will also play a role in these observations).

This motif consisting of two genes with feedback loops is found at the core of several known decision-making architectures throughout biology, but of course, it will not exist in isolation in real biological systems (although theoretical studies have underlined how analysis of its dynamics can be valuable in understanding its downstream physiological significance ${ }^{8,44,47}$ ). However, our hypothesised link between energy variability and decision-making dynamics is not restricted to this motif. Relaxing the bistable picture used here, links between energy variability and cell fate are anticipated in a broad variety of other cell decisions. One pertinent example is the formation of bacterial persister phenotypes in the presence of antibiotics. One regulatory motif involved here is the hipBA module, which expresses HipA (toxin) and HipB (antitoxin), which can form a complex that represses their own expression. Rotem et al. ${ }^{87}$ found that cells become dormant if the level of HipA exceeds a threshold, and as the level of HipA exceeded the threshold further, it determined the duration of dormancy. This is similar to our model and architecture where the protein levels, $x_{1}$ and $x_{2}$, feed back into the system and establish the generated cell fate; we would then predict that intracellular energy budget is a factor involved in the persister decision. Further connections between intracellular energy budget and the persister cell decision have been observed in both S. aureus ${ }^{31}$ and E. coli ${ }^{59}$. T-cell fate decisions in immunology provide another example. After an antigenic stimulation, a T-cell switches from a resting catabolic state (naïve and memory T-cells) to an anabolic effector $\mathrm{T}$-cell state which requires more energy and grows and proliferates ${ }^{88-90}$. Once activated, effector T-cells differentiate into different lineages, each containing a distinctive phenotype, including functional properties and metabolic changes ${ }^{91-94}$. In relation to our model, we predict that any ATP differences between cells (for example, arising through variability in metabolic poise ${ }^{91,95}$ ) will affect the expression levels and interaction dynamics of specific master regulators (including mTOR, cytokines, GATA3 and T-bet ${ }^{94,96-99}$ ), modulating the lineage to which an effector T-cell transitions.

We predict that ATP variability will influence fate decisions across this broad variety of systems, organisms and branches of life. Generally, the propensity to choose different cell fates will be intrinsically affected by ATP supply (in addition to extrinsic signals that may also be linked to nutrient and energy availability), and for bistable regulatory motifs, higher ATP levels will promote undifferentiated states from which more decisions are possible.

The influence of noise in gene expression ${ }^{52-54}$ on the behaviour and stability of this system will also be valuable to link to specific biological situations. Together, the inclusion of noise and additional genetic actors will help shed 
further light on how decisions are made by the cell (in our model, how transitions between available attractor states are accomplished). Some subtle and re-entrant behaviour was observed in the bifurcation dynamics associated with transitions between different numbers of distinct stable states. If this behaviour is general across other architectures, it may provide a potential optimum intracellular energy range for phenotypic diversity, enabling a cell to have superior adaption to stochastic extracellular environmental change.

We hope that this study has opened a new line of enquiry in the current understanding of cellular decision making. Prior to this work, energy variability in cells had, to our knowledge, not been considered as a key determinant of the cellular decision-making landscape. Through consideration of the potential effect of energy variability on intracellular physiology, understanding the mechanisms behind cellular decision-making may improve. We hope that further knowledge on how intracellular energy budget changes a cell's decision-making landscape could be a step towards understanding the fundamental mechanisms behind cellular decision-making and towards developing novel methods to either promote or inhibit the consequences.

\section{Data availability}

All scripts used for this study are openly accessible through: https://github.com/StochasticBiology/energyvariability-decision-making.

Received: 27 June 2019; Accepted: 12 December 2019;

Published online: 27 December 2019

\section{References}

1. Cortijo, S., Aydin, Z., Ahnert, S. \& Locke, J. C. Widespread inter-individual gene expression variability in Arabidopsis thaliana. Molecular systems biology 15, e8591 (2019).

2. Kalmar, T. et al. Regulated fluctuations in nanog expression mediate cell fate decisions in embryonic stem cells. PLoS biology 7 , e1000149 (2009).

3. Ozbudak, E. M., Thattai, M., Kurtser, I., Grossman, A. D. \& Van Oudenaarden, A. Regulation of noise in the expression of a single gene. Nature genetics 31, 69 (2002).

4. Blake, W. J. et al. Phenotypic consequences of promoter-mediated transcriptional noise. Molecular cell 24, 853-865 (2006).

5. Fraser, D. \& Kaern, M. A chance at survival: gene expression noise and phenotypic diversification strategies. Molecular microbiology 71, 1333-1340 (2009).

6. Bowsher, C. G. \& Swain, P. S. Environmental sensing, information transfer, and cellular decision-making. Current opinion in biotechnology 28, 149-155 (2014).

7. Balázsi, G., van Oudenaarden, A. \& Collins, J. J. Cellular decision making and biological noise: from microbes to mammals. Cell 144, 910-925 (2011).

8. Johnston, I. G. \& Bassel, G. W. Identification of a bet-hedging network motif generating noise in hormone concentrations and germination propensity in Arabidopsis. Journal of The Royal Society Interface 15, 20180042 (2018).

9. Mitchell, J., Johnston, I. G. \& Bassel, G. W. Variability in seeds: biological, ecological, and agricultural implications. Journal of experimental botany 68, 809-817 (2016).

10. Topham, A. T. et al. Temperature variability is integrated by a spatially embedded decision-making center to break dormancy in Arabidopsis seeds. Proceedings of the National Academy of Sciences 114, 6629-6634 (2017).

11. Easwaran, H., Tsai, H.-C. \& Baylin, S. B. Cancer epigenetics: tumor heterogeneity, plasticity of stem-like states, and drug resistance. Molecular cell 54, 716-727 (2014).

12. Chang, H. H., Hemberg, M., Barahona, M., Ingber, D. E. \& Huang, S. Transcriptome-wide noise controls lineage choice in mammalian progenitor cells. Nature 453, 544 (2008).

13. Wernet, M. F. et al. Stochastic spineless expression creates the retinal mosaic for colour vision. Nature 440, 174 (2006).

14. Waddington, C. H. The Strategy of the Genes. (Allen \& Unwin, London, 1957).

15. Park, I.-H. et al. Reprogramming of human somatic cells to pluripotency with defined factors. Nature 451, 141 (2008).

16. Teslaa, T. \& Teitell, M. A. Pluripotent stem cell energy metabolism: an update. The EMBO journal 34, 138-153 (2015).

17. Takahashi, K. et al. Induction of pluripotent stem cells from adult human fibroblasts by defined factors. cell 131, 861-872 (2007).

18. Mahla, R. S. Stem cells applications in regenerative medicine and disease therapeutics. International journal of cell biology 2016 (2016).

19. Hughes, B. S., Cullum, A. J. \& Bennett, A. F. An experimental evolutionary study on adaptation to temporally fluctuating pH in Escherichia coli. Physiological and Biochemical Zoology 80, 406-421 (2007).

20. Levy, S. F. Cellular heterogeneity: benefits besides bet-hedging. Current Biology 26, R355-R357 (2016).

21. Mitchell, A. et al. Adaptive prediction of environmental changes by microorganisms. Nature 460, 220 (2009).

22. Acar, M., Mettetal, J. T. \& Van Oudenaarden, A. Stochastic switching as a survival strategy in fluctuating environments. Nature genetics 40, 471 (2008).

23. Süel, G. M., Garcia-Ojalvo, J., Liberman, L. M. \& Elowitz, M. B. An excitable gene regulatory circuit induces transient cellular differentiation. Nature 440, 545 (2006).

24. Smits, W. K., Kuipers, O. P. \& Veening, J.-W. Phenotypic variation in bacteria: the role of feedback regulation. Nature Reviews Microbiology 4, 259 (2006).

25. Newman, J. R. et al. Single-cell proteomic analysis of S. cerevisiae reveals the architecture of biological noise. Nature 441,840 (2006).

26. Phillips, Z. \& Strauch, M. Bacillus subtilis sporulation and stationary phase gene expression. Cellular and Molecular Life Sciences CMLS 59, 392-402 (2002).

27. Andersson, A., Ronner, U. \& Granum, P. E. What problems does the food industry have with the spore-forming pathogens Bacillus cereus and Clostridium perfringens? International journal of food microbiology 28, 145-155 (1995).

28. Wells-Bennik, M. H. et al. Bacterial spores in food: survival, emergence, and outgrowth. Annual review offood science and technology 7, 457-482 (2016).

29. Balaban, N. Q., Merrin, J., Chait, R., Kowalik, L. \& Leibler, S. Bacterial persistence as a phenotypic switch. Science 305, 1622-1625 (2004).

30. Cohen, N. R., Lobritz, M. A. \& Collins, J. J. Microbial persistence and the road to drug resistance. Cell host \& microbe 13, 632-642 (2013).

31. Conlon, B. P. et al. Persister formation in Staphylococcus aureus is associated with ATP depletion. Nature microbiology $\mathbf{1}, 16051$ (2016).

32. Kussell, E., Kishony, R., Balaban, N. Q. \& Leibler, S. Bacterial persistence: a model of survival in changing environments. Genetics 169, 1807-1814 (2005).

33. Fauvart, M., De Groote, V. N. \& Michiels, J. Role of persister cells in chronic infections: clinical relevance and perspectives on antipersister therapies. Journal of medical microbiology 60, 699-709 (2011). 
34. Lewis, K. Persister cells. Annual review of microbiology 64, 357-372 (2010).

35. Mulcahy, L. R., Burns, J. L., Lory, S. \& Lewis, K. Emergence of Pseudomonas aeruginosa strains producing high levels of persister cells in patients with cystic fibrosis. Journal of bacteriology 192, 6191-6199 (2010).

36. Zhang, Y., Yew, W. W. \& Barer, M. R. Targeting persisters for tuberculosis control. Antimicrobial agents and chemotherapy 56, 2223-2230 (2012).

37. Karlebach, G. \& Shamir, R. Modelling and analysis of gene regulatory networks. Nature Reviews Molecular Cell Biology 9, 770 (2008).

38. Das Neves, R. P. et al. Connecting variability in global transcription rate to mitochondrial variability. PLoS biology 8, e1000560 (2010).

39. Kafri, M., Metzl-Raz, E., Jona, G. \& Barkai, N. The cost of protein production. Cell reports 14, 22-31 (2016).

40. Yaginuma, H. et al. Diversity in ATP concentrations in a single bacterial cell population revealed by quantitative single-cell imaging. Scientific reports 4, 6522 (2014)

41. Takaine, M., Ueno, M., Kitamura, K., Imamura, H. \& Yoshida, S. Reliable imaging of ATP in living budding and fission yeast. J. Cell Sci. jcs-230649 (2019).

42. Yoshida, T., Kakizuka, A. \& Imamura, H. Bteam, a novel bret-based biosensor for the accurate quantification of ATP concentration within living cells. Scientific reports 6, 39618 (2016).

43. De Col, V. et al. ATP sensing in living plant cells reveals tissue gradients and stress dynamics of energy physiology. Elife 6, e26770 (2017).

44. Johnston, I. G. et al. Mitochondrial variability as a source of extrinsic cellular noise. PLoS computational biology 8, e1002416 (2012).

45. Katajisto, P. et al. Asymmetric apportioning of aged mitochondria between daughter cells is required for stemness. Science 348, 340-343 (2015).

46. Schieke, S. M. et al. Mitochondrial metabolism modulates differentiation and teratoma formation capacity in mouse embryonic stem cells. Journal of Biological Chemistry 283, 28506-28512 (2008).

47. Huang, S., Guo, Y.-P., May, G. \& Enver, T. Bifurcation dynamics in lineage-commitment in bipotent progenitor cells. Developmental biology 305, 695-713 (2007).

48. Nilsson, T., Schultz, V., Berggren, P.-O., Corkey, B. E. \& Tornheim, K. Temporal patterns of changes in ATP/ADP ratio, glucose 6-phosphate and cytoplasmic free $\mathrm{Ca}^{2+}$ in glucose-stimulated pancreatic beta-cells. Biochemical journal 314, 91 (1996).

49. Jensen, P. R. \& Michelsen, O. Carbon and energy metabolism of ATP mutants of Escherichia coli. Journal of bacteriology 174, 7635-7641 (1992).

50. Stitt, M., Lilley, R. M. \& Heldt, H. W. Adenine nucleotide levels in the cytosol, chloroplasts, and mitochondria of wheat leaf protoplasts. Plant physiology 70, 971-977 (1982).

51. Jones, E., Oliphant, T., Peterson, P., \& others. (2001). SciPy: Open source scientific tools for Python. Retrieved from http://www. scipy.org/.

52. Paulsson, J. Summing up the noise in gene networks. Nature 427, 415 (2004).

53. Pedraza, J. M. \& van Oudenaarden, A. Noise propagation in gene networks. Science 307, 1965-1969 (2005).

54. Thattai, M. \& Van Oudenaarden, A. Intrinsic noise in gene regulatory networks. Proceedings of the National Academy of Sciences 98 , 8614-8619 (2001).

55. Feng, H. \& Wang, J. A new mechanism of stem cell differentiation through slow binding/unbinding of regulators to genes. Scientific reports 2, 550 (2012).

56. Huang, S. Non-genetic heterogeneity of cells in development: more than just noise. Development 136, 3853-3862 (2009).

57. Okawa, S., Nicklas, S., Zickenrott, S., Schwamborn, J. C. \& del Sol, A. A generalized gene-regulatory network model of stem cell differentiation for predicting lineage specifiers. Stem cell reports 7, 307-315 (2016).

58. Zhou, J. X. \& Huang, S. Understanding gene circuits at cell-fate branch points for rational cell reprogramming. Trends in genetics 27, 55-62 (2011).

59. Shan, Y. et al. ATP-dependent persister formation in Escherichia coli. MBio. 8, e02267-16 (2017).

60. Bokes, P., King, J. R., Wood, A. T. \& Loose, M. Transcriptional bursting diversifies the behaviour of a toggle switch: hybrid simulation of stochastic gene expression. Bulletin of mathematical biology 75, 351-371 (2013).

61. Cherry, J. L. \& Adler, F. R. How to make a biological switch. Journal of theoretical biology 203, 117-133 (2000).

62. Lipshtat, A., Loinger, A., Balaban, N. Q. \& Biham, O. Genetic toggle switch without cooperative binding. Physical review letters 96, 188101 (2006).

63. Loinger, A., Lipshtat, A., Balaban, N. Q. \& Biham, O. Stochastic simulations of genetic switch systems. Physical Review E 75, 021904 (2007).

64. Perez-Carrasco, R., Guerrero, P., Briscoe, J. \& Page, K. M. Intrinsic noise profoundly alters the dynamics and steady state of morphogen-controlled bistable genetic switches. PLoS computational biology 12, e1005154 (2016).

65. Tyson, J. J., Chen, K. C. \& Novak, B. Sniffers, buzzers, toggles and blinkers: dynamics of regulatory and signaling pathways in the cell. Current opinion in cell biology 15, 221-231 (2003).

66. Verd, B., Crombach, A. \& Jaeger, J. Classification of transient behaviours in a time-dependent toggle switch model. BMC systems biology 8, 43 (2014)

67. Warren, P. B. \& ten Wolde, P. R. Chemical models of genetic toggle switches. The Journal of Physical Chemistry B 109, 6812-6823 (2005).

68. Ackers, G. K., Johnson, A. D. \& Shea, M. A. Quantitative model for gene regulation by lambda phage repressor. Proceedings of the National Academy of Sciences 79, 1129-1133 (1982).

69. Atsumi, S. \& Little, J. W. A synthetic phage $\lambda$ regulatory circuit. Proceedings of the National Academy of Sciences 103, 19045-19050 (2006).

70. Aurell, E. \& Sneppen, K. Epigenetics as a first exit problem. Physical review letters 88, 048101 (2002).

71. Ptashne, M. A genetic switch: phage lambda revisited 3 (Cold Spring Harbor Laboratory Press Cold Spring Harbor, NY, 2004).

72. Niwa, H. et al. Interaction between oct3/4 and cdx2 determines trophectoderm differentiation. Cell 123, 917-929 (2005).

73. Xiong, W. \& Ferrell, J. E. Jr. A positive-feedback-based bistable 'memory module' that governs a cell fate decision. Nature 426, 460 (2003).

74. Muto, A. et al. Bach2 represses plasma cell gene regulatory network in b cells to promote antibody class switch. The EMBO journal 29, 4048-4061 (2010).

75. Al-Radhawi, M. A., Del Vecchio, D. \& Sontag, E. D. Multi-modality in gene regulatory networks with slow promoter kinetics. PLoS computational biology 15, e1006784 (2019).

76. Andrecut, M., Halley, J. D., Winkler, D. A. \& Huang, S. A general model for binary cell fate decision gene circuits with degeneracy: indeterminacy and switch behavior in the absence of cooperativity. PLoS One 6, e19358 (2011).

77. Becskei, A., Séraphin, B. \& Serrano, L. Positive feedback in eukaryotic gene networks: cell differentiation by graded to binary response conversion. The EMBO journal 20, 2528-2535 (2001).

78. Folguera-Blasco, N., Pérez-Carrasco, R., Cuyàs, E., Menendez, J. A. \& Alarcón, T. A multiscale model of epigenetic heterogeneitydriven cell fate decisionmaking. PLoS computational biology 15, e1006592 (2019).

79. Wu, F., Su, R.-Q., Lai, Y.-C. \& Wang, X. Engineering of a synthetic quadrastable gene network to approach Waddington landscape and cell fate determination. ELife 6, e23702 (2017). 
80. Bokes, P., King, J. R. \& Loose, M. A bistable genetic switch which does not require high co-operativity at the promoter: a twotimescale model for the PU. 1-GATA-1 interaction. Mathematical medicine and biology: a journal of the IMA 26, 117-132 (2009).

81. Duff, C., Smith-Miles, K., Lopes, L. \& Tian, T. Mathematical modelling of stem cell differentiation: the PU. 1-GATA-1 interaction. Journal of mathematical biology 64, 449-468 (2012).

82. Roeder, I. \& Glauche, I. Towards an understanding of lineage specification in hematopoietic stem cells: a mathematical model for the interaction of transcription factors GATA-1 and PU. 1. Journal of theoretical biology 241, 852-865 (2006).

83. Tian, T. \& Smith-Miles, K. Mathematical modeling of gata-switching for regulating the differentiation of hematopoietic stem cell. In BMC systems biology, vol. 8, S8 (BioMed Central, 2014).

84. Ezashi, T., Das, P. \& Roberts, R. M. Low $\mathrm{O}_{2}$ tensions and the prevention of differentiation of hES cells. Proceedings of the National Academy of Sciences 102, 4783-4788 (2005).

85. Chung, S. et al. Mitochondrial oxidative metabolism is required for the cardiac differentiation of stem cells. Nature Reviews Cardiology 4, S60 (2007).

86. Armstrong, L. et al. Human induced pluripotent stem cell lines show stress defense mechanisms and mitochondrial regulation similar to those of human embryonic stem cells. Stem cells 28, 661-673 (2010).

87. Rotem, E. et al. Regulation of phenotypic variability by a threshold-based mechanism underlies bacterial persistence. Proceedings of the National Academy of Sciences 107, 12541-12546 (2010)

88. Bental, M. \& Deutsch, C. Metabolic changes in activated t cells: an nmr study of human peripheral blood lymphocytes. Magnetic resonance in medicine $29,317-326$ (1993).

89. Jones, R. G. \& Thompson, C. B. Revving the engine: signal transduction fuels T cell activation. Immunity 27, 173-178 (2007).

90. Pearce, E. L. Metabolism in T cell activation and differentiation. Current opinion in immunology 22, 314-320 (2010).

91. MacIver, N. J., Michalek, R. D. \& Rathmell, J. C. Metabolic regulation of T lymphocytes. Annual review of immunology 31, 259-283 (2013).

92. Wan, Y. Y. \& Flavell, R. A. How diverse-CD4 effector T cells and their functions. Journal of molecular cell biology 1, 20-36 (2009).

93. Zhu, J., Yamane, H. \& Paul, W. E. Differentiation of effector CD4 T cell populations. Annual review of immunology 28, 445-489 (2009).

94. Evans, C. M. \& Jenner, R. G. Transcription factor interplay in T helper cell differentiation. Briefings in functional genomics 12, 499-511 (2013).

95. Hirahara, K. et al. Mechanisms underlying helper T-cell plasticity: implications for immune-mediated disease. Journal of Allergy and Clinical Immunology 131, 1276-1287 (2013).

96. Araki, K. et al. mtor regulates memory CD8 T-cell differentiation. Nature 460, 108 (2009).

97. Düvel, K. et al. Activation of a metabolic gene regulatory network downstream of mTOR complex 1. Molecular cell 39, 171-183 (2010).

98. Zeng, H. et al. mTORC1 and mTORC2 kinase signaling and glucose metabolism drive follicular helper T cell differentiation. Immunity 45, 540-554 (2016).

99. Zhou, L., Chong, M. M. \& Littman, D. R. Plasticity of CD4+ T cell lineage differentiation. Immunity 30, 646-655 (2009).

\section{Acknowledgements}

R.K. acknowledges $\mathrm{PhD}$ funding from the Wellcome Trust (grant reference 108876/Z/15/Z). This project has received funding from the European Research Council (ERC) under the European Union's Horizon 2020 research and innovation programme (Grant agreement No. 805046 (EvoConBiO) to I.G.J). I.G.J. also acknowledges a Turing Fellowship from the Alan Turing Institute. S.J. thanks the BBSRC for grant code BB/M021386/1.

\section{Author contributions}

S.J. and I.G.J. conceived and supervised the study; R.K. wrote the simulation code and performed the mathematical analysis; all authors contributed to writing the manuscript.

\section{Competing interests}

The authors declare no competing interests.

\section{Additional information}

Supplementary information is available for this paper at https://doi.org/10.1038/s41598-019-56587-5.

Correspondence and requests for materials should be addressed to I.G.J.

Reprints and permissions information is available at www.nature.com/reprints.

Publisher's note Springer Nature remains neutral with regard to jurisdictional claims in published maps and institutional affiliations.

(c) (i) Open Access This article is licensed under a Creative Commons Attribution 4.0 International License, which permits use, sharing, adaptation, distribution and reproduction in any medium or format, as long as you give appropriate credit to the original author(s) and the source, provide a link to the Creative Commons license, and indicate if changes were made. The images or other third party material in this article are included in the article's Creative Commons license, unless indicated otherwise in a credit line to the material. If material is not included in the article's Creative Commons license and your intended use is not permitted by statutory regulation or exceeds the permitted use, you will need to obtain permission directly from the copyright holder. To view a copy of this license, visit http://creativecommons.org/licenses/by/4.0/.

(c) The Author(s) 2019 